\title{
The Efficacy of Anti-Retroviral Therapy on HIV Patients with and Without Tuberculosis: A Comparative Study in a State Hospital Bida Niger State Nigeria
}

\author{
Ebenezer Obi Daniel $^{1, ~}{ }^{*}$, Aminat Abiola Ojewale ${ }^{1}$, Paul Olaiya Abiodun ${ }^{1}$, Gabriel Omoniyi Ayeni ${ }^{1}$, \\ Olayinka Victor Ojo ${ }^{1}$, Oladapo Michael Olagbegi ${ }^{2}$
}

${ }^{1}$ Department of Public Health School of Public Health, Texila American University Georgetown, Guyana, South America

${ }^{2}$ Department of Physiotherapy School of Health Scienczes, University of Kwazulu-Natal Westville Campus, Durban, South Africa

Email address:

dannypressy@yahoo.com (E. O. Daniel)

*Corresponding author

\section{To cite this article:}

Ebenezer Obi Daniel, Aminat Abiola Ojewale, Paul Olaiya Abiodun, Gabriel Omoniyi Ayeni, Olayinka Victor Ojo, Oladapo Michael Olagbegi. The Efficacy of Anti-Retroviral Therapy on HIV Patients with and Without Tuberculosis: A Comparative Study in a State Hospital Bida Niger State Nigeria. International Journal of HIV/AIDS Prevention, Education and Behavioural Science.

Vol. 5, No. 2, 2019, pp. 97-104. doi: 10.11648/j.ijhpebs.20190502.13

Received: July 22, 2019; Accepted: August 13, 2019; Published: August 26, 2019

\begin{abstract}
There is a general broad knowledge surrounding the possible complications and opportunistic infections that patients with HIV can be exposed to, one of which is Tuberculosis, a popular co-morbid disease in HIV patients. Several studies have unraveled the efficacy of ARVs on the CD4 counts in HIV patients, but there exists knowledge gap in establishing the efficacy of ARV therapy among HIV patients with Tuberculosis (TB). The main objective of this study was to compare the efficacy of antiretroviral therapy on HIV positive patients with and without tuberculosis. A quasi-experimental design was employed in this study. Eighty (80) patients were recruited in total from the ARV-TB clinic of general hospital Bida. Forty (40) HIV patients without TB, and HIV patients with TB were recruited consecutively into group A and B respectively. Each group participants' baseline CD4 counts were estimated and recorded, after which both groups were subjected to a six weeks ARV therapy. Data were collected weekly (for six weeks) by conducting laboratory test of CD4 counts for both groups' patients. The data were analyzed using SPSS version 25.0 software. Descriptive statistics of mean, standard deviation and percentages were used to summarize the data, while inferential statistics of t-test, ANOVA, ANCOVA and Bonferoni pairwise comparison were used to test the significant differences as appropriate. Alpha level was set at 0.05 . The study revealed that the CD4 count of HIV patients without TB (group A) at baseline was found to be statistically significantly higher than those with TB (group B), and also revealed a statistical significant difference in the CD 4 count across the seven-time-point period of the study (baseline and the six weeks of ARV therapy) in the two groups $(\mathrm{P}<0.05)$. The study also revealed that there was no statistically significant difference in the CD4 count of groups A and B comparatively after six weeks of ARV therapy. Recommendations among others include; extension of the study tentacle to increased number of health facilities and longer study period, conducting studies on the impact of the dual therapy (ARV and anti-TB) on the co-infected patients, and the need for all stakeholders involved in the treatment of HIV patients to continuously update their knowledge base on the management of the HIV patients with and without TB, for a more productive and goal-oriented treatment outcome, void of sentiments and discrimination.
\end{abstract}

Keywords: HIV, Tuberculosis, Antiretroviral Therapy 


\section{Introduction}

\subsection{Human Immunodeficiency Virus (HIV)}

A lentivirus responsible for HIV infection and overtime without any intervention leads to Acquired immunedeficiency Disease syndrome (AIDs). An individual with HIV has compromised immunity and are prone to several opportunistic infections especially tuberculosis. Average survival time after being infected with HIV without any intervention is between 9-11 years [1]. Acquisition of HIV infection is majorly through sexual contact, others are sharing of sharps, vertical transmission from mother to child, blood transfusion and others.

HIV infection majorly affects the CD4 T-cell, untreated $\mathrm{HIV}$ reduces the number of $\mathrm{CD} 4$ cell in the body leading to damage of the immune system, making it harder for the body to fight off infection and some other disease, [2] hence the need for monitoring infected patient CD4-cells, other cells affected by HIV are the macrophages and dendritic cells. There are two types which are HIV1 and HIV2.

The HIV epidemic in Nigeria is $3.1 \%$ estimated prevalence among the age group 15-45 years in 2015 with 180,000 estimated deaths among these group and 828,867 estimated adults on antiretroviral drugs [3].

\subsection{Tuberculosis}

Tuberculosis (TB) on the other hand is a bacterial infection caused by the exposure to Mycobacterium Tuberculosis with primary focus on the lungs called pulmonary TB and can also affect other parts of the body outside lungs referred to as extra-pulmonary TB [4]. Tuberculosis occurs in two forms viz: latent TB and full blow (Active) TB disease and close to one - third of the world population has dormant (latent) and many with latent $\mathrm{TB}$ may live without progressing to active $\mathrm{TB}$, others who has latent TB with compromised immunity can progress to full blow (Active) disease [5]. TB is acquired through inhalation of the $M$. tuberculosis release into air by an infected person through coughing, sneezing or vigorous speaking. Although not all the people with TB become sick, those with latent TB are not sick until the latent phase become active disease due to compromised immunity which for example in cases of HIV infection [6], the presence of HIV increases the risk of latent TB becoming disease compare to the others without HIV infection, other conditions with low immunity that can put an individual at risk of developing TB disease or progression of latent TB to full disease are diabetics, malignancies and others.

Patient with TB often presents with one or more of the following symptoms: Fever, Weight Loss, Excessive Night Sweat, Cough of more than 2weeks, Chest pain, Body weakness and Production of bloody sputum. But in most people living with HIV co-infected with TB usually presents with few or less classic TB symptoms, they are therefore frequently having what is called "sub-clinical" TB which is often unrecognized as TB and subsequently delay both the diagnosis and treatment [7].
$\mathrm{HIV} / \mathrm{TB}$ co-infection is the presence of the two diseases in one person, a condition refers to as double burden disease and an individual with such condition is regarded to be having an AIDs defining illness, most time such individuals may not present with cough, hence need for high level of suspicion of TB in HIV positive clients. TB/HIV co-infection reduces patient lifespan there is therefore need for active case finding of TB among the people living with HIV as well as among the general population in order to reduce the risk of exposure among people with low immunity.

The use of Highly Active Antiretroviral Therapy (HAART) show association of low incidents of TB across all the strata of socio-economic status. Number of TB cases averted among the HIV positive client by the use of HAART was greatest among HIV patients with WHO stage 3 and 4 and in those with CD4 count less than 200cells. HAART reduces the incidence of HIV/TB coinfection by $>80 \%$ in the endemic area with TB/HIV infection, the proactive effect of HAART was more in symptomatic patients and those with advance immune suppression [8].

There are cases of HIV co-infected with TB with few cases of co-infected multi-drug resistance tuberculosis (MDR-TB) in Bida local government area of Niger state, Nigeria, most of these cases are among those HIV positive patients who are not regular on clinic visit as well as not HAART drug compliance or those presented lately. The main significance of this study is its tendency to serve as a clue of information on how to provide effective and efficient treatment for HIV patients with and without TB in an individualized manner. It will serve as a guiding tool for good therapeutic intervention in such category of clients.

The latest estimates from the World Health Organization (WHO 2019) show that progress has been made towards the target. Global TB deaths among people living with HIV have fallen by $42 \%$ since 2010 , from 520000 down to 300000 in 2017. However, the estimates also indicate that progress remains uneven and further efforts are needed to address the main challenges, including the need for equity and ensuring that vulnerable groups have access to integrated HIV and TB services. In 2017, five low- or middle-income countries had already achieved or exceeded the target of a $75 \%$ reduction in TB deaths among people living with HIV-India (84\%), Eritrea $(83 \%)$, Djibouti $(78 \%)$, Malawi $(78 \%)$ and Togo (75\%). A further 18 countries reduced TB deaths among people living with HIV by more than $50 \%$ and are on track to achieve the target by the end of 2020 , provided that scale-up of services is maintained [9].

\section{Method}

A quasi experimental design was employed in this study. The study aimed at comparing the antiretroviral efficacy (using the pre- and post-experimental CD4 count to assess the ART efficacy) among the HIV positive patients with TB, and those HIV positive patient without TB, who are accessing treatment at the ART/TB unit of the General Hospital, Bida, Niger State in Nigeria. This study was 
conducted at the ART/TB unit of the General Hospital, Bida. The Ethical Approval for the study was obtained from the Health Research and Ethics Committee of the hospital, and the quality improvement facility team of Bida state hospital before commencing the study and the cover letter containing the details of the study was submitted at the administrative unit of the hospital for permission to carry out the research. Informed consent was obtained from individual participants with the details of the research explained to them and promised to maintain confidentiality and only those consented were involved in the study.

This study employed purposeful sampling method in recruiting the participants among the adult HIV positive client with or without TB who are clinically stable (using clinical symptoms) and immunologically stable who are accessing treatment in the facility. Forty (40) patients were purposively selected each from among the co-infected (HIVTB) patients, and HIV patients without TB. Both age and sex matching were ensured to reduce the effect of the confounding variables.

Data were collected using laboratory analysis results at baseline and post-therapy period of weekly and after six weeks. The CD4 count machine is valid and of high sensitivity. The data were analyzed using SPSS 25.0 version software (SPSS Inc., Chicago, Illinois, USA). Descriptive statistics of mean, standard deviation and percentages were used to summarize the data. The groups' age and baseline CD4 count were compared using independent t-test while the categorical variable (sex) was compared using a chi-square test. A one-way repeated measures ANOVA was computed for analysis within group time effects on CD4 count while Bonferroni post-hoc multiple pairwise comparison was used to locate time intervals that significantly differ for each group. Since the two groups were significantly different in their baseline CD4 count, One-way Analysis of Covariance (ANCOVA) was used for between group effects of ARV treatment with baseline CD4 count as a covariate. Cohen's rule of thumb (0.01- small, 0.06 - moderate, 0.14 - large) was used to categorize effect sizes (partial eta-squared) while level of significance was set at $\mathrm{p}<0.05$.

\section{Results}

\subsection{Participants}

Eighty patients (male: 21 ; female: 59 ) with HIV on antiretroviral therapy ( 40 without $\mathrm{TB}, 40$ with $\mathrm{TB}$ ) participated in the study. They were allocated to treatment groups A and B based on co-morbidity of TB. Their age ranged 20 to 50 years with mean value of $33.74 \pm 7.97$ years.

\subsection{Comparison of the Groups' Demographic Variables and Baseline CD4 Count}

The groups' demographic variables and baseline CD4 count are compared in Table 1. Independent t-test and Chisquare test respectively showed that there was no significant difference $(p>0.05)$ between groups A and B regarding their age $(p=0.813)$ and sex $(p=0.204)$, but patients with HIV in Group A had significantly higher mean baseline CD4 count $(\mathrm{p}<0.001)$ than those in Group B.

Table 1. Comparison of participants'demographic variables and baseline CD4 count.

\begin{tabular}{llll}
\hline \multirow{2}{*}{ Variable } & Group A & Group B & T \\
\cline { 2 - 4 } & Mean \pm SD & Mean \pm SD & 0.237 \\
\hline Age & $33.95 \pm 7.79$ & $33.53 \pm 8.24$ & p-value \\
Baseline CD4 Count & $409.03 \pm 186.53$ & $278.95 \pm 70.22$ & 0.813 \\
\hline
\end{tabular}

\begin{tabular}{lllllll}
\hline Sex & Frequency & \% & Frequency & \% & Chi-square & p-value \\
\hline Male & 8 & 20.0 & 13 & 32.5 & 0.204 \\
Female & 32 & 80.0 & 27 & 67.5 & 1.614 \\
\hline
\end{tabular}

*denotes significance at $\mathrm{p}<0.05$

SD: Standard deviation

Group A: Patients with HIV on ARV without TB

Group B: Patients with HIV on ARV with TB

\subsection{Within Group Comparison of CD4 Count of Participants in Group A (Patients with HIV on ARV Without TB) at the Seven Time Points of the Study}

The results of repeated measures ANOVA used for within group (time effect) analysis of the CD4 count of participants in Group A is presented in Table 2. Repeated measures ANOVA showed significant differences in CD4 count across the seven time points of the study for participants in Group A (MS = $35975.87, \mathrm{~F}=6.010, \mathrm{p}<0.001)$ with large effect size (0.134). Table 3 shows the results of post hoc analysis done using
Bonferroni multiple pairwise comparisons, the results indicated significant increase $(\mathrm{p}<0.005)$ in CD4 of the participants at baseline/week 1, baseline/week 2, baseline/week 3, baseline/week 4 , baseline/week 6 , week $2 /$ week 4 and week 3/week 4 time intervals of the study. The trend of CD4 count changes across the seven time points of the study for participants in Group A is illustrated in Figure 1. There was marked increase in participants' CD4 count between baseline and week 1, but it fluctuated between week 1 and week 5 and later increased from week 5 to the end of the study. 
Table 2. Within group comparison of CD4 count of participants in Group A (Patients with HIV on ARV without TB) at the seven time-points of the study.

\begin{tabular}{llllll}
\hline Variable & Mean \pm SD & $\mathbf{9 5 \%}$ CI & & MS & F \\
\hline CD4 Bas & $409.03 \pm 186.53$ & 349.37 & 468.68 & 35975.87 & 6.010 \\
CD4 WK 1 & $444.43 \pm 192.01$ & 383.02 & 505.83 & & \\
CD4 WK 2 & $443.80 \pm 192.85$ & 382.12 & 505.48 & & \\
CD4 WK 3 & $445.53 \pm 193.56$ & 383.62 & 507.43 & & \\
CD4 WK 4 & $447.38 \pm 193.77$ & 385.40 & 509.35 & & \\
CD4 WK 5 & $442.05 \pm 193.61$ & 380.13 & 503.97 & & \\
CD4 WK 6 & $453.68 \pm 205.68$ & 387.89 & 519.46 & & \\
\hline
\end{tabular}

*denotes significance at $\mathrm{p}<0.05$

MS: mean square, ES: Effect size (partial eta-square)

Bas: Baseline, WK: Week, SD: Standard deviation, CI: Confidence Interval

Table 2 above showed significant differences in CD4 count across the seven time points of the study for participants in Group A (HIV patients on ARV without TB).

Table 3. Bonferroni post-hoc multiple pairwise analysis of time effect on CD4 count of Group A participants.

\begin{tabular}{|c|c|c|}
\hline Time Interval & Mean Difference & p-value \\
\hline Baseline vs Week 1 & 35.400 & $<0.001^{*}$ \\
\hline Baseline vs Week 2 & 34.775 & $<0.001 *$ \\
\hline Baseline vs Week 3 & 36.500 & $<0.001 *$ \\
\hline Baseline vs Week 4 & 38.350 & $<0.001 *$ \\
\hline Baseline vs Week 5 & 33.025 & 0.092 \\
\hline Baseline vs Week 6 & 44.650 & $0.041 *$ \\
\hline Week 1 vs Week 2 & -0.625 & 1.000 \\
\hline Week 1 vs Week 4 & 2.950 & 0.107 \\
\hline Week 1 vs Week 5 & -2.375 & 1.000 \\
\hline Week 1 vs Week 6 & 9.250 & 1.000 \\
\hline Week 2 vs Week 3 & 1.725 & 0.071 \\
\hline Week 2 vs Week 4 & 3.575 & $<0.001 *$ \\
\hline Week 2 vs Week 5 & -1.750 & 1.000 \\
\hline Week 2 vs Week 6 & 9.875 & 1.000 \\
\hline Week 3 vs Week 6 & 8.150 & 1.000 \\
\hline Week 4 vs Week 5 & -5.325 & 1.000 \\
\hline Week 4 vs Week 6 & 6.300 & 1.000 \\
\hline Week 5 vs Week 6 & 11.625 & 1.000 \\
\hline
\end{tabular}

*denotes significance at $\mathrm{p}<0.05$

Table 3 above revealed that there was a significant increase in CD4 count of the participants in group A at baseline/week 1, baseline/week 2, baseline/week 3, baseline/week 4, baseline/week 6, week 2/week 4 and week 3/week 4-time intervals of the study.

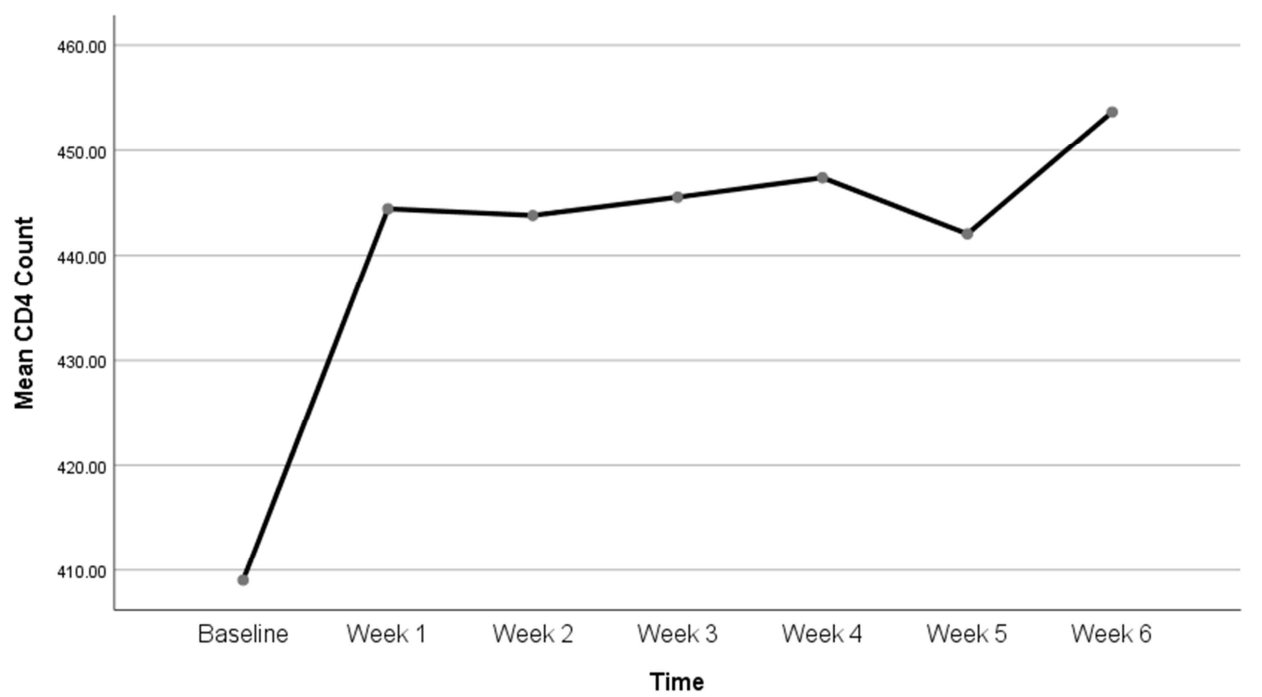

Figure 1. Trends of CD4 count of participants in Group A (Patients with HIV on ARV without TB) across the seven time-points of the study. 
Figure 1 above indicated a marked increase in participants' (group A) CD4 count between baseline and week 1, but it fluctuated between week 1 and week 5 and later increased from week 5 to the end of the study.

\subsection{Within Group Comparison of CD4 Count of Participants in Group B (Patients with HIV on ARV with TB) at the Seven Time Points of the Study}

The results of repeated measures ANOVA used for within group (time effect) analysis of the CD4 count of participants in Group B is presented in Table 4. The results indicated significant differences in CD4 count across the seven time points of the study for participants in Group B (MS $=15029.64, \mathrm{~F}=$ $26.554, \mathrm{p}<0.001)$ with large effect size $(0.405)$. Table 5 shows the results of post hoc analysis computed using Bonferroni multiple pairwise comparisons, the results showed significant increase in CD4 of the participants at baseline/week 1, baseline/week 2, baseline/week 3, baseline/week 4, baseline/week 5 , baseline/week 6 , week 1 /week 6 and week $2 /$ week 5 , week $2 /$ week 6 , week $3 /$ week 5 , week $3 /$ week 6 , week $4 /$ week 5 , week $4 /$ week 6 , and week $5 /$ week 6 time intervals of the study.

The trend of CD4 count changes across the seven time points of the study participants in Group B is displayed in Figure 2.

There was marked increase in participants' CD4 count between baseline and week 1 which slightly increased between weeks 1 and 2 . It however showed a slim decrease between weeks 2 and 3 and later increased steadily till the end of the study.

Table 4. Within group comparison of CD4 count of participants in Group B (Patients with HIV on ARV with TB) at the seven time-points of the study.

\begin{tabular}{|c|c|c|c|c|c|c|c|}
\hline Variable & Mean \pm SD & $95 \% \mathrm{CI}$ & & MS & $\mathbf{F}$ & p-value & ES \\
\hline CD4 Bas & $278.95 \pm 70.22$ & 256.49 & 301.41 & 15029.64 & 26.554 & $<0.001^{*}$ & 0.405 \\
\hline CD4 WK 1 & $299.48 \pm 66.44$ & 278.23 & 320.72 & & & & \\
\hline CD4 WK 2 & $300.41 \pm 69.05$ & 278.34 & 322.51 & & & & \\
\hline CD4 WK 3 & $300.18 \pm 66.87$ & 278.79 & 321.56 & & & & \\
\hline CD4 WK 4 & $301.48 \pm 67.09$ & 280.02 & 322.93 & & & & \\
\hline CD4 WK 5 & $304.73 \pm 7.22$ & 283.23 & 326.22 & & & & \\
\hline CD4 WK 6 & $306.63 \pm 67.75$ & 284.96 & 328.29 & & & & \\
\hline
\end{tabular}

*denotes significance at $\mathrm{p}<0.05$

MS: mean square, ES: Effect size (partial eta-square) Bas: Baseline, WK: Week, SD: Standard deviation, CI: Confidence Interval

From Table 4 above, it can be inferred that there were significant differences in CD4 count across the seven time points of the study for participants in Group B.

Table 5. Bonferroni post-hoc multiple pairwise analysis of time effect on CD4 count of Group B participants.

\begin{tabular}{|c|c|c|c|}
\hline Time Interval & Mean Difference & & p-value \\
\hline Baseline vs Week 1 & $20.525^{*}$ & 4.156 & $<0.001^{*}$ \\
\hline Baseline vs Week 2 & $21.475^{*}$ & 4.540 & $0.001 *$ \\
\hline Baseline vs Week 3 & $21.225^{*}$ & 4.522 & $0.001 *$ \\
\hline Baseline vs Week 4 & $22.525^{*}$ & 4.423 & $<0.001^{*}$ \\
\hline Baseline vs Week 5 & $25.775^{*}$ & 4.332 & $<0.001^{*}$ \\
\hline Baseline vs Week 6 & $27.675^{*}$ & 4.311 & $<0.001^{*}$ \\
\hline Week 1 vs Week 2 & 0.950 & 1.317 & 1.000 \\
\hline Week 1 vs Week 3 & 0.700 & 1.461 & 1.000 \\
\hline Week 1 vs Week 4 & 2.000 & 1.586 & 1.000 \\
\hline Week 1 vs Week 5 & 5.250 & 1.569 & $0.038^{*}$ \\
\hline Week 1 vs Week 6 & 7.150 & 1.657 & $0.002 *$ \\
\hline Week 2 vs Week 3 & -0.250 & 0.624 & 1.000 \\
\hline Week 2 vs Week 4 & 1.050 & 0.834 & 1.000 \\
\hline Week 2 vs Week 5 & 4.300 & 0.948 & $0.001 *$ \\
\hline Week 2 vs Week 6 & 6.200 & 1.132 & $<0.001^{*}$ \\
\hline Week 3 vs Week 4 & 1.300 & 0.544 & 0.460 \\
\hline Week 3 vs Week 5 & 4.550 & 0.728 & $<0.001 *$ \\
\hline Week 3 vs Week 6 & 6.450 & 0.991 & $<0.001 *$ \\
\hline Week 4 vs Week 5 & 3.250 & 0.328 & $<0.001^{*}$ \\
\hline Week 4 vs Week 6 & 5.150 & .583 & $<0.001 *$ \\
\hline Week 5 vs Week 6 & 1.900 & .345 & $<0.001^{*}$ \\
\hline
\end{tabular}

*denotes significance at $\mathrm{p}<0.05$

Table 5 above showed significant increase in CD4 of the participants in group B at baseline/week 1, baseline/week 2, baseline/week 3, baseline/week 4, baseline/week 5, baseline/week 6 , week $1 /$ week 6 and week $2 /$ week 5 , week $2 /$ week 6 , week $3 /$ week 5 , week $3 /$ week 6 , week $4 /$ week 5 , week $4 /$ week 6 , and week $5 /$ week 6 time intervals of the study. 
From figure 2 indicated that there was marked increase in participants' (group B) CD4 count between baseline and week 1 which slightly increased between weeks 1 and 2 . It further showed a slim decrease between weeks 2 and 3 and later increased steadily till the end of the study.

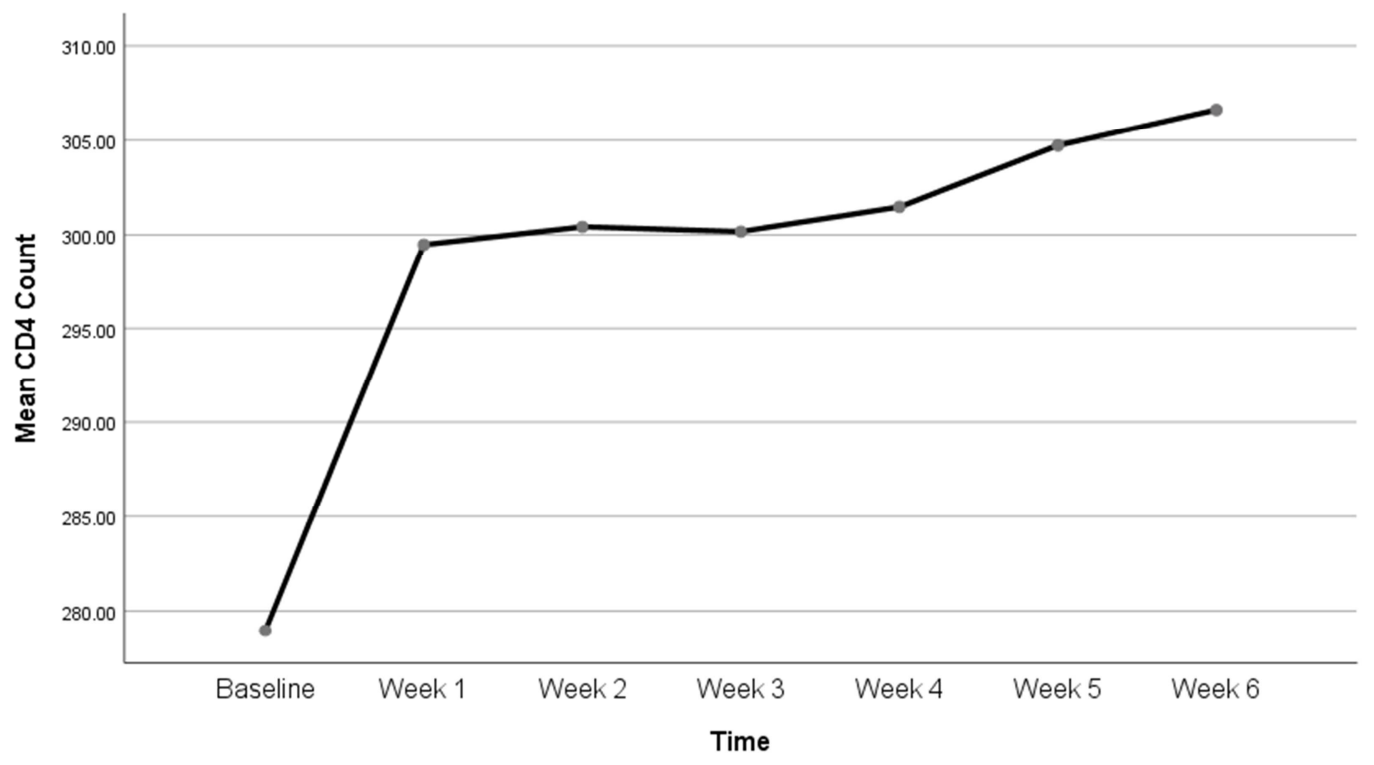

Figure 2. Trends of CD4 count of participants in Group B (Patients with HIV on ARV with TB) across the seven time-points of the study.

\subsection{Between Group Comparisons of Changes in CD4 Count of Participants in Groups $A$ and $B$ at the End of the Study}

Between-group comparison of the groups' changes in CD4 count during the course of the study was compared and presented in Table 6. One-way ANCOVA (computed using baseline value as a covariate) did not indicate any significant difference $(\mathrm{MS}=5499.979, \mathrm{~F}=1.361, \mathrm{p}=0.247$ ) in the groups' changes in CD4 count at the end of the study.

Table 6. ANCOVA between group comparisons of changes in CD4 count of participants in Groups A and B at the end of the study.

\begin{tabular}{llllll}
\hline Variable & Mean \pm SD & Mean \pm SD & MS & F & p-value \\
\hline CD4 Bas & $409.03 \pm 186.53$ & $278.95 \pm 70.22$ & 5499.979 & 1.361 & 0.247 \\
CD4 WK 6 & $453.68 \pm 205.68$ & $306.63 \pm 67.75$ & & & 0.017 \\
\hline
\end{tabular}

Note: Covariates appearing in the model are evaluated at the following values: CD4 Baseline $=343.9875$.

*denotes significance at $\mathrm{p}<0.05$

MS: mean square, ES: Effect size (partial eta-square)

Bas: Baseline, WK: Week, SD: Standard deviation, CI: Confidence Interval

Table 6 above did not reveal any significant difference in the CD4 count changes at the end of the study, when groups A and B were compared

\subsection{Percentage Changes in the Groups' (A and B) CD4 Count Between Baseline and End of Study}

Percentage changes in the groups' CD4 count between baseline and week 6 (the end of study) were calculated using the formula:

$$
\% \text { Change }=\frac{\mathrm{CD} 4 \text { count at week } 6-\mathrm{CD} 4 \text { count at baseline }}{\mathrm{CD} 4 \text { count at baseline }} \times 100 \%
$$

The results showed that participants in Group A (patients with HIV on ARV without TB) had percentage increase of $12.98 \pm 17.73 \%$ in their CD4 count while Group B participants (patients with HIV on ARV with TB) demonstrated increase of $10.95 \pm 9.63 \%$ in their CD4 count from baseline to the end of the study.

\section{Discussion}

In this study, (Eighty) 80 participants who are confirmed
HIV positive on antiretroviral therapy with or without tuberculosis were recruited of which there were 21 males $(26.25 \%)$ and 59 females $(73.75 \%)$. They were allocated into treatment group A and B based on their co-morbidity with 40 participants who are HIV positive without TB in group A and 40 who are HIV positive with TB in group B. Their age ranged between 20 to 50 years with mean value of $33.74 \pm$ 7.97 years. with the mean age of group A (HIV without TB) of $33.95 \pm 7.79$ and that of group B (HIV with TB) of 33.53 \pm 8.24 . These groups' demographic variable result showed 
that there was no significant difference and most of the participants are females $(73.75 \%)$ within the reproductive age group. This age model is in line with the similar study findings done in IMO State, Nigeria by Duru C.B et.al [10]. This study showed that Group A (HIV without TB) had statistically significantly higher mean baseline CD4 count ( $p$ $<0.001$ ) than those in Group B (HIV co-infected with TB), this could be due to the dual disease burden effect of the HIV and tuberculosis in group B. TB co-infection with HIV commonly occur at low CD4 count in HIV positive patients as seen in the work done by Nwabuko. et.al [11] which showed an inverse relationship between CD4 count and the occurrence of tuberculosis of HIV patient co-infected with $\mathrm{TB}$ and for the fact that co-infected patients has slower rate of CD4 recovery when compare with HIV patients without TB as reported by Penelope et.al [12] Hence, the alternate hypothesis (H1) that stated that there will be significant difference in the baseline value of CD4 count of HIV patients with or without TB on ARV was accepted while the null hypothesis is rejected because there was statistically significant difference in the baseline value of CD4 count of group A compare to that of group B, as revealed in this study.

The result of this study also revealed statistically significant different at seven-time points of the study (from baseline to 6 weeks post-therapy) within-group comparison of CD4 count of participants in group A and B, with further confirmation using post hoc analysis. This statistically significant difference in the CD4 counts at seven-time points, within each group, may be due to the fact that the HIV patients in each group maintain an appreciable degree of drug-therapy compliance in the study period. This finding is in line with the work done by Sten Skogmer [13] which showed significant increase/difference in the CD4 count patients with HIV/TB co-infection after the commencement of anti-TB, which may be due to possible reversible effect of active TB on CD4 cell hemostasis leading to increase in CD4 count after the commencement of therapy. However, it does not agree with the findings of Wejse et.al [14] which show no significant increase/difference in CD4 count of co-infected HIV/TB patients on drugs after eight months of study. Also, the Sten Skogmer [13] work done in Ethiopia showed significant increase in CD4 count of both HIV/TB coinfected and TB patients on drugs. Hence, the alternate hypothesis which stated that there will be statistically significant difference in CD4 counts of HIV patients with and without TB within weeks of ART therapy was accepted and the null hypothesis is rejected.

Another finding of this study showed that there was no statistically significant difference in the pre and post therapy CD4 counts when groups A and B were compared. Even though the pre and post-therapy CD4 counts' percentage comparison results showed that participants in Group A (patients with HIV on ARV without TB) had percentage increase of $12.98 \pm 17.73 \%$ in their CD4 count while Group B participants (patients with HIV on ARV with TB) demonstrated increase of $10.95 \pm 9.63 \%$ in their CD4 count from baseline to the end of the study, implying a slight CD4 count increment in group A above group B, after six weeks of ARV therapy, however, the significant difference could not be established statistically. This revelation may be as result of possible optimal drug compliance in both groups coupled with the fact that HIV/TB co-infected patients are both on ART and anti-tuberculosis therapy (serving as augmenting therapy), which make them to have almost the same rate of CD4 increase with HIV patients without TB. Hence it is possible therefore that the dual effect of ART and anti-TB drugs makes group B CD4 counts improved in a similar pace with that of group A during the study period. This result is in concordance with the findings of a similar research done in Cape Town by Richard. et.al which compared HIV/TB coinfected patients on ART and anti-TB drugs (in one group) with those HIV/T [15] B co-infected on only anti-TB drug without ART (in another group) The result of their study showed a significant increase in the CD4 count of those coinfected on both ART and anti-TB when compare with CD4 count of those co-infected on only anti-TB drug. Based on the findings of this study, we failed to reject the null hypothesis which stated that there will be no significant difference in the post-therapy CD4 count of HIV patients with or without TB after six weeks of ART and the alternate hypothesis is here rejected.

\section{Conclusion}

Although, this study finding showed statistically significant difference in the baseline CD4 count of HIV patients without TB when compared with that of HIV patients with TB, and statistically significant difference of CD4 count in both groups within weeks of the study period, but pre-therapy (baseline) and post-therapy (after 6 weeks) findings showed no significant CD4 count difference between the two groups. This implies that, with proper management and good drug compliance on the part of the patients, same treatment result is probably achievable in both groups.

\section{Recommendations}

Based on the findings of this study, it is recommended that;

1. The tentacle of the study should be extended to other geopolitical zones in Nigeria with a greater number of $\mathrm{ARV} / \mathrm{TB}$ health facilities involved, as the present study was done in the North Central and limited to only one ARV health facilities.

2. Further study should take into consideration the effect of diet on both group A (HIV without TB) and group B (HIV with TB), as it might have served as a confounding variable in this present study

3. More advanced studies on the impact of the combined therapy of ARV and anti-TB on the CD4 counts of coinfected (HIV with TB) patients should be considered to establish more facts in that regard.

4. Since this study was conducted in one of the Sub- 
Saharan African countries (Nigeria), a future comparative study with what is obtainable in the developed nations will be more knowledge-enriching.

5. It will be more clinically productive for all the health staff and other stakeholders involved in the treatment of HIV patients to be updating their knowledge on how best to manage this category of clients knowing full well that they are prone to opportunistic infections like TB

\section{References}

[1] UNAIDS (2007): AIDS Epidemic update.

[2] Centre for Disease Control-CDC (2018): New HIV infection/Ending AIDS.

[3] UNAIDS (2016): Global Report.

[4] World Health Organization- WHO (2009): Global Tuberculosis Report.

[5] Stop TB Partnership \& World Health Organization. (2006). The global plan to stop TB, 2006-2015 / Stop TB Partnership. World Health Organization. https://apps.who.int/iris/handle/10665/43404.

[6] CDC (2016): STD Prevention Conference report World Health Organization-WHO (2017): Tuberculosis - World Health Organization https//www.who.int/news-room/factsheets/detail/tuberculosis.

[7] Penelope K. Ellis, Willam J. Martin, Peter J. Dodd (2017): CD4 count and tuberculosis risk in HIV-positive adults not on ART: A systematic review and meta-analysis PeerJ 5 (2): e4165 DOI: 10. 7717/peerj. 4165.

[8] Motasm Badri, Douglas Wilson, Prof. Robin Wood (2002) Effect of Highly Active Antiretroviral on Incidence of Tuberculosis in South Africa, ScienceDirect (THE LANCET, Vol. 359, Issue 9323, June 2002.
[9] Joint United Nations Programme on HIV/AIDS (UNAID) (2019) Tuberculosis and HIV: progress towards the 2020 target.

[10] Duru C. B, Uwakwe K. A, Diwe K. C, Nnebue C. C, Chineke H. N, Emerole C. A (2014): Prevalence of Active TB Among HIV Positive Patients Attending A Teaching Hospital in Imo State, South East, Nigeria: A 6years Review (2002-2012), Indian Journal of Medical Research and Pharmaceutical Science.

[11] Nwabuko C. O, Ejele O. A, Chuku A., Nnoli M. A, Chukwuonye I. I, (2012), Prevalence of Tuberculosis-HIV Coinfection and Relationship between TB and CD4/ESR in HIV patients in Niger Delta Region of Nigeria, IORS Journal of Dental and Medical Sciences (JDMS), vol. 2 (4): 01-04. www.iosrjournal.org.

[12] Penelope K. EllisWillam J. MartinPeter J. Dodd (2017): CD4 count and tuberculosis risk in HIV-positive adults not on ART: A systematic review and meta-analysis. PeerJ 5 (2): e4165 DOI: $10.7717 /$ peerj. 4165 .

[13] Sten Skogmer, Thomas S., Taye T. B., Zalalem H. J, Gudeta T., Jonas B., Per Bjorkman, (2013), CD4 Cell Level During Treatment for Tuberculosis in Ethiopia Adult and Clinical Markers Associated with CD4 Lymphocytopenia, PLOS PATHOGENS. https://doi.org/10.1371/journal.pone.0083270.

[14] Wejse C., Furtado A., Camara C., Luneborg-Nielsen M., Sodemann M., Gerstoft J., Katzenstein T. L., (2013): Impact of Tuberculosis Treatment on CD4 cell count, HIV RNA and P24 Antigen in Patients with HIV and Tuberculosis. Science Direct, vol. 17 (10): e907-e912.

[15] Richard Hayes, Tazeem Bhatia, Jamie Enoch, Mishal Khan, Sophie Mathewson, David Heymann,, Osman Dar (2018): Setting targets for HIV/AIDS-What lessons can be learned from other disease control programmes? 2019 https://doi.org/10.1371/journal.pmed.1002735. 